\title{
Vesicular systems for delivering conventional small organic molecules and larger macromolecules to and through human skin
}

Article

Accepted Version

El Maghraby, G. M. and Williams, A. C. (2009) Vesicular systems for delivering conventional small organic molecules and larger macromolecules to and through human skin. Expert Opinion on Drug Delivery, 6 (2). pp. 149-163. ISSN 1742-5247 doi: https://doi.org/10.1517/17425240802691059 Available at https://centaur.reading.ac.uk/4457/

It is advisable to refer to the publisher's version if you intend to cite from the work. See Guidance on citing.

To link to this article DOI: http://dx.doi.org/10.1517/17425240802691059

Publisher: Informa

All outputs in CentAUR are protected by Intellectual Property Rights law, including copyright law. Copyright and IPR is retained by the creators or other copyright holders. Terms and conditions for use of this material are defined in the End User Agreement. 


\section{CentAUR}

Central Archive at the University of Reading

Reading's research outputs online 
Vesicular systems for delivering conventional small organic molecules and larger macromolecules to and through human skin

\begin{abstract}
The history of using vesicular systems for drug delivery to and through skin started nearly three decades ago with a study utilizing phospholipid liposomes to improve skin deposition and reduce systemic effects of triamcinolone acetonide. Subsequently, many researchers evaluated liposomes with respect to skin delivery, with the majority of them recording localized effects and relatively few studies showing transdermal delivery effects. Shortly after this, Transfersomes were developed with claims about their ability to deliver their payload into and through the skin with efficiencies similar to subcutaneous administration. Since these vesicles are ultradeformable, they were thought to penetrate intact skin deep enough to reach the systemic circulation. Their mechanisms of action remain controversial with diverse processes being reported. Parallel to this development, other classes of vesicles were produced with ethanol being included into the vesicles to provide flexibility (as in ethosomes) and vesicles were constructed from surfactants and cholesterol (as in niosomes). Thee ultradeformable vesicles showed variable efficiency in delivering low molecular weight and macromolecular drugs. This article will critically evaluate vesicular systems for dermal and transdermal delivery of drugs considering both their efficacy and potential mechanisms of action.
\end{abstract}

\title{
Keywords
}

Ethosomes; Liposomes; Niosomes; Transfersomes; Transdermal Drug Delivery; Macromolecular Drugs

\section{Introduction}


The skin is the largest single organ in the body and so provides formulators with a large surface area for drug application. Transdermal drug delivery has many potential advantages over other routes of administration. These include the avoidance of gastrointestinal tract problems and hepatic first pass effects and improvement in patient compliance. Unfortunately, the barrier nature of skin presents difficulties for delivering many drugs into and through it [1]. Various approaches have been utilized to improve transdermal delivery [2-3]. These include the use of chemical penetration enhancers [4], optimization of chemical potential of the drug, for example by increasing the driving force through supersaturation [5-6], electrically driving molecules into or through the tissue employing iontophoresis [7], physically disrupting the skin structure, for example by electroporation or sonophoresis [8-9] or incorporation of the drug in microemulsions [10-11]. Vesicular drug delivery systems such as Liposomes, Niosomes, Ethosomes and Transfersomes provide an alternative for improved drug delivery to and through the skin [12-17].

Variable functions have been reported for vesicular systems as skin drug delivery systems [18]. They can provide a localized depot in the skin and reduce the amounts of drug permeating into the systemic circulation thus minimizing the unwanted effects. They may also provide targeted delivery through the appendageal pathway (hair follicles and sweat ducts). Additionally, vesicles can enhance transdermal drug delivery, increasing systemic drug concentrations. Indeed, some vesicles may possess several of the above functions, with the main effect depending on the type of vesicle as well as the application protocol. This diversity becomes greater when considering the mechanisms of action of these nano-structures as skin drug delivery systems. Accordingly, the aim of 
this article is to provide a critical review of vesicles as skin drug delivery systems with emphasis on the types of vesicles as well as the nature of the encapsulated drug.

\section{Body}

\subsection{Types of vesicular systems}

Alternative terminology has been used to describe vesicular systems but all researchers agree that they are of a similar morphology but with different functions and/or compositions [13-16].

Liposomes are vesicles in which one or more lipid bilayer(s) entrap an aqueous volume. Their major components are usually phospholipids with or without cholesterol. The stratum corneum lipid liposomes (SCLL) are vesicular systems made of lipids with a composition similar to the lipids found in the outer layer of human skin, the stratum corneum. Transfersomes (ultradeformable vesicles) are structurally similar to liposomes but they differ in function (see below); again phospholipids are the major components but an additional surfactant acts as an edge activator to modify elasticity and increase deformability. Ethosomes are phospholipid vesicles, which include ethanol to increase elasticity, whereas niosomes comprise surfactants together with cholesterol and may include small proportions of phospholipids.

\subsection{Vesicular skin drug delivery of conventional small organic molecules}

Vesicular skin delivery of such compounds could produce localized effect, deliver the drug into or through the skin appendages or provide transdermal delivery with 
increased systemic effects. These effects are considered below with a critical appraisal of the relevant literature.

\subsubsection{Localized effects}

Steroidal drugs in liposomes have been extensively studied; the localizing effect of liposomes was recorded for steroids in the first ever report on vesicles as skin drug delivery systems [12]. In this work, dipalmitoylphosphatidylcholine (DPPC) and cholesterol $(\mathrm{CH})$ vesicles increased the deposition of triamcinolone acetonide in the epidermis and dermis, and reduced percutaneous absorption compared with a standard ointment [12]. Incorporating the same formulation in a gel dosage form, similar findings were observed relative to a gel containing free drug and the components of liposomes at the same concentrations [19]. These initial findings reflected the importance of liposomal encapsulation and hence good entrapment efficiency of the drug in the vesicle for efficient skin drug delivery. Similar findings were reported for progesterone and econazole delivered from liposomes with a similar lipid composition [20]. In contrast, the topical input of $5 \alpha$-dihydrotestosterone from similar vesicles was inferior to that from an acetone solution containing the same drug concentration when assessed by monitoring the size of the flank organs of the female hamster [21]. This contradiction was attributed to four possible factors; first, the use of different steroids; second, the first group monitored delivery by measuring skin deposition whilst the later studies measured a biological effect; then, the studies used different animals and finally, the schemes of application were different. An alternative explanation is that both groups used equal drug concentrations rather than equal thermodynamic activity for their controls and that the 
biological effect on the hamster flank organs may require systemic drug delivery rather than a liposome-mediated localized effect. The deposition of hydrocortisone into human skin was significantly higher after application of phosphatidylcholine (PC)/CH liposomes compared with an emulsion ointment form. The improved skin deposition and reduced systemic effects were further confirmed by monitoring blanching effects and determining the pharmacokinetic parameters of hydrocortisone [22]. Similarly, SCLL and phospholipid vesicles showed better skin deposition of corticosteroids with the former being superior. The anti-inflammatory effects from nano-structural delivery paralleled the skin accumulation results [23].

In contrast to the above, it was reported that liposomal encapsulation facilitated both the retention and permeation of triamcinolone acetonide compared with an ointment formulation [24]. This discrepancy could be due to the use of different membranes; permeability differences between species and especially the relatively poor correlation seen between animal skin models and human skin can make direct comparisons between laboratory protocols problematic.

In addition to steroids, the localizing effects of liposomes have also been sought for treating various skin conditions such as psoriasis. Thus, radiotracer studies in mice revealed improved skin accumulation of tacrolimus from a vesicle-containing lotion compared with intravenous injection of a solution or the liposomal formulation [25]. In addition to the 9-fold increase in skin levels of the drug following topical nano-aggregate delivery compared with systemic delivery, the vesicular formulation also prevented delayed-type hypersensitivity reactions seen with systemic provision. 
Local anesthetics are another group of low molecular weight drugs for which liposomal encapsulation was researched for dermal and transdermal delivery. Using the pin-prick assay, prolonged anesthesia from tetracaine-containing liposomes was shown with a cream control formulation being ineffective [26]. Using the same assay, liposomes prolonged lidocaine anesthesia compared with a conventional cream with a higher deposition in the epidermis and dermis being recorded [27]. The improved anesthetic effect after vesicular delivery could be thus attributed to improved skin accumulation. In another report, liposomal tetracaine increased both drug permeation through, and deposition into, human skin compared to an ointment containing the same drug concentration [28]. The anesthesia recorded after liposomal application was even stronger and deeper than that obtained from a commercial eutectic mixture of local anesthetics (EMLA, $2.5 \%$ lidocaine and 2.5\% prilocaine) in humans [29-30].

The above studies all employed liposomes made of phospholipids or skin lipids. Planas et al (1992) reported an improved anesthetic effect of lidocaine and tetracaine from Transfersomes. In vivo studies employed rats and humans to assess anesthesia after topical application of Transfersomes (PC plus sodium cholate), liposomes (PC) and drug solution [31]. It is important to note that the authors applied the tested formulations under occlusion for 25 minutes, which is against the recommended open application protocol. Transfersomes produced enhanced anesthesia compared with drug solution or traditional liposomes. Surprisingly, topically-applied anesthetic Transfersomes generated an effect equivalent to that created after subcutaneous injection of the same formulation.

The skin delivery of 5-fluorouracil (5-FU) from similar Transfersomes was investigated after application of a finite dose with saturated aqueous solution used as the 
control [32]. The study employed aqueous or water/ethanol (50\% v/v) receptor solutions. The results (Figure 1) showed a marginal increase in transepidermal delivery into the aqueous receptor. However, the ethanolic receptor significantly increased drug permeation when using the vesicles. These findings were taken as evidence for improved deposition of the drug in the skin as ethanol is expected to diffuse into the tissue disrupting deposited vesicles and extracting the drug. The skin distribution from Transfersomes was described as dose dependent [33]; the use of finite or infinite doses can thus affect the relative proportion of localized to transdermal drug amounts and hence optimization of the applied dose is an important facet of Transfersomal action.

\section{Insert Figure 1}

Elastic niosomes of Tween 61 and cholesterol were recently developed by incorporating ethanol at $25 \%$. These vesicles were successful in delivering the antiinflammatory drug diclofenac diethylammonium into and through the skin when compared to non-elastic niosomes which increased the deposition of the drug only [34]. The improved transdermal effect could have resulted from the enhancing effect of ethanol.

\subsubsection{Targeted delivery to skin appendages}

The above literature determined localizing effects of vesicular systems on the basis of increased drug deposition into the stratum corneum and viable epidermis. Other workers have studied the potential of such nano-structures for targeting the appendages, especially to the pilosebaceous units (hair follicles with their associated sebaceous 
glands). This area was extensively reviewed by Lauer et al (1996) and Lauer (1999) [3536].

Employing the hamster ear model, liposomes of $\mathrm{PC}, \mathrm{CH}$ and phosphatidylserine (PS) delivered the fluorescent hydrophilic dye, carboxyfluorescein, into the pilosebaceous units. They were more efficient than aqueous solutions even after incorporation of $10 \%$ ethanol or $0.05 \%$ sodium lauryl sulphate, or using propylene glycol as the donor vehicle [37-38].

Targeted delivery of cimetidine into the pilosebaceous glands and other skin strata of the Syrian male hamster ear was recorded after topical application of the drug in $50 \%$ aqueous ethanol, niosomes, or in liposomes. Monitoring the anti-androgenic effect, the first two formulations were pharmacologically active with the later being ineffective. The authors explained the lack of a biological effect from the phospholipid vesicles, despite increased drug provision into the tissue, on the basis that the negative charge of lipids can inactivate the drug by forming an ion pair with it at $\mathrm{pH} 5.5$ (the $\mathrm{pH}$ of the formulation) [39]. Autoradiography revealed the presence of considerable amounts of caffeine in the appendages after topical application of liposomes to rat skin but most of the drug was localized in the epidermis [40].

In contrast to the above reports, neither liposomes nor mixed micelles provided any advantage over an ethanolic gel with regard to follicular delivery of isotretinoin. This finding was attributed to the highly lipophilic nature of the drug which would intrinsically target the sebaceous gland [41]. However, the use of an ethanolic preparation as the control may be responsible for possible misleading results since ethanol is capable of enhancing the follicular delivery through partial solubilisation of the 
sebum or softening of the material in the duct, which could result in the ethanolic control producing significant drug input. The control was thus shown to be equivalent to the other formulations. Whilst these findings could suggest a positive targeting effect of liposomes and mixed micelles, we can only conclude that they were as effective as the ethanolic gel.

Vesicular preparations were found to be superior in the treatment of acne vulgaris compared with conventional preparations including alcoholic lotions [42-43]. This study provides strong evidence for effective vesicular targeting to skin appendages.

Further, in vitro permeation through hamster flank skin and in vivo deposition in hamster ear recently demonstrated the potential of liquid-state liposomes and niosomes for successfully delivering finasteride to the pilosebaceous unit [44].

\subsubsection{Improved transdermal delivery}

Despite concentrating on the localizing effect of liposomes with improved drug deposition into skin and its appendages, some early reports recorded improved transdermal delivery from these nano-aggregates. After topical application of finite doses of liposomes to hairless mouse skin in vitro, it was reported that vesicles can provide greater permeation of lipophilic drugs compared to an aqueous solution [45]. Formulations containing unilamellar soya-lecithin/CH liposomes advanced the percutaneous absorption of methyl nicotinate compared with an aqueous solution or gel formulations [46]. Vesicles containing Epikuron 200, a phospholipid with unsaturated alkyl side chains (fluid liposomes), produced high percutaneous absorption and tissue 
distribution rather than skin accumulation. Renal elimination of inulin was 20-fold higher after usage of such liposomes compared to delivery from aqueous solution [47].

While researchers were reporting mainly localized and rarely transdermal effects of liposomes, Cevc and Blume (1992) [13] claimed that Transfersomes can penetrate intact to the deep layers of the skin and may progress far enough to reach the systemic circulation. Importantly, they recommended that, for successful delivery, Transfersomes must be applied under non-occlusive conditions, although a deviation from this protocol can be found [31] where improved anesthesia was reported after occluded treatment with anesthetic Transfersomes. Transfersomes improved the regio-specificity and the biological activity of the corticosteroids hydrocortisone, dexamethasone and triamcinolone acetonide, in vivo. The effect depended on the applied dose and it was concluded that this carrier can target the drug into the viable skin and, when used in a higher dose, can distribute the medicament throughout the body [33]. Transfersomes provided suppression of arachidonic acid-induced oedema with an efficiency equivalent to a lotion containing 5-times the drug concentration of that in deformable vesicles, after 0.5 hour. Subsequently, after 2 hours, the Transfersome formulation was more efficacious than the lotion. Evaluating standard liposomes, no oedema suppression was found after 0.5 hour. After 2 hours, however, liposomes produced a measurable suppression. The effect of liposomes (after 2 hours) was about one third that of deformable vesicles and about half that of the lotion (with 5-times more drug). The authors stated that the late effect of the vesicle formulation arose from free drug permeation following its release from liposomes [33]. However, if this explanation is valid, vesicles would be expected to provide one fifth of the efficacy of the lotion 
(containing free drug), unless there is some penetration enhancing effect for such liposomes.

In addition, the arachidonic acid-induced oedema suppression test (acute murine ear oedema model) was used to evaluate the anti-inflammatory effect of $\mathrm{Cu}, \mathrm{Zn}$ superoxide dismutase after topical application in Transfersomes, mixed micelles or liposomes. Of all the tested carriers only Transfersomes significantly reduced oedema [48].

In a series of investigations involving an optimized experimental design, estradiol skin delivery from a variety of ultradeformable and standard liposomes was investigated [49]. The previously optimized ultradeformable formulations employed various edge activators (surfactants); PC with sodium cholate (UD1), PC with Span 80 (UD2) and PC with Tween 80 (UD3) [50]. The standard vesicles encompassed pure PC vesicles (non rigid liposomes, SL1), PC with $\mathrm{CH}$ (membrane stabilized, SL2), and two rigid liposomes of DPPC (SL3) and DPPC/CH (SL4). The studies involved a low dose open application of the formulations to human epidermal membrane hydrated by an "open hydration" protocol that maintained the transepidermal water gradient and evaluated both the transdermal flux and skin deposition. The results (Figure 2) indicated that all types of liposomes improved both estradiol deposition into and permeation through the epidermis compared with the saturated aqueous control. The ultradeformable vesicles were better than the standard liposomes with respect to transepidermal drug flux but there were no significant differences within the different types of nano-carriers with regard to estradiol accumulation in the skin. The ultradeformable liposomes reduced the time of maximum 
flux $\left(\mathrm{T}_{\max }\right)$ by $11-16 \%$. For the standard liposomes, $\mathrm{T}_{\max }$ was either constant (SL1) or increased by $10-20 \%$ (SL2-4).

\section{Insert Figure 2}

The incorporation of a surfactant in liposomes increases the fluidity (flexibility) or elasticity of the lipid bilayers [51]. Accordingly, it can be concluded that flexible liposomes are more efficient in delivering drugs across the epidermis. The presence of surfactants (edge activators) was considered responsible for vesicle deformability, which allows for improved transdermal drug delivery [52-54]. The incorporation of ethanol in lipid vesicles (Ethosomes) is an alternative approach to fluidize the lipid membrane and thus enhance drug provision [16-17]. Also, flexible niosomes showed higher delivery efficiencies compared to rigid niosomes [55-56].

\subsection{Vesicular skin delivery of large molecular weight compounds}

Recently, the potential of vesicles to deliver large molecules into and through the skin has been extensively investigated recently, for molecules ranging from large molecular weight peptides to DNA based vaccines. As for the small organic molecules, vesicular skin delivery can provide localized effects, deliver the drug into or through the skin appendages or promote transdermal delivery with increased systemic effects.

\subsubsection{Localized effect}

Topical delivery of the peptide drug interferon from liposomes was superior to that from a water-in-oil emulsion or aqueous solution as revealed by reduced lesion 
scores from a cutaneous herpes simplex virus guinea pig model. Interferon delivery from SCLL produced greater remission of lesions compared to the input from phospholipid liposomes. With respect to the method of nano-aggregate preparation, those produced by a dehydration rehydration (DRV) method were more effective than when prepared by mechanical shaking or extrusion [57]. The authors attributed the superiority of DRV liposomes to increased drug partitioning into the vesicle, but the advantage could also have been due to increased entrapment efficiency. In a subsequent study the in vitro tape stripping of guinea pig skin was used to determine the skin deposition of interferon, with the amount of drug permeated also evaluated [58]. Liposomes increased the deposition of interferon into stratum corneum and deeper skin strata with no drug detected in the receptor. Again SCLL prepared by DRV were considered superior to other formulations. This study indicated that the previously obtained pharmacological effect was due to localization of the drug [57]. The deposition of cyclosporin-A into the stratum corneum of hairless mice was increased, whilst transdermal absorption reduced, from various liposomal formulations compared to drug solutions. The formulations were ranked with respect to drug localization as SCLL > phospholipid liposomes > O/W emulsion (emulsifier composition similar to lipid content of phospholipid vesicles) > hydroalcoholic drug solution (5 mg/ml in $40 \%$ ethanol) [59]. This series of investigations emphasized the localizing effects of liposomes and highlighted the dependence of deposition efficiency on the lipid composition and method of preparation, though it should be noted that the animal models used may not reflect delivery into human skin.

\subsubsection{Targeted delivery to skin appendages}


Liposomal skin deposition of $\gamma$-interferon was monitored in humans, hairless mice and hamster skin in vitro. Vesicles were superior over an aqueous solution containing the same amount of the drug. The greatest accumulation was recorded in hamster skin, which possesses the highest follicular density. It was thus suggested that the follicular pathway could be a route for macromolecular drug deposition from liposomes [60].

\subsubsection{Improved transdermal delivery}

Qualitative immunohistochemical examination revealed that liposomeencapsulated antibodies (molecular weight 20000-50000 Da) distributed rapidly into the deep cutaneous regions of piglet skin in vivo, compared with no penetration after application of aqueous solution or empty vesicles. Quantitative studies using radiolabelled markers revealed not only improved deposition into the deep strata but also clearly increased transdermal delivery from vesicles compared with aqueous solution [6162]. A liposomal formulation of $\mathrm{PC}$ and $\mathrm{CH}$ augmented the uptake of biologically active recombinant human $\gamma$-interferon into the epidermis of viable human skin compared with aqueous solution [63].

Successful systemic delivery of insulin has been achieved by Transfersomes as reported from in vivo mice and human studies. Drug distribution studies confirmed the transfer of ${ }^{125}$ I-insulin across intact skin with subsequent distribution throughout the body after topical application of Transfersomes, and pharmacodynamic studies revealed a reduction in the blood glucose levels. The efficiency from the vesicles was comparable to that obtained after S.C. injection of the same preparation but with a longer lag time. This lag time may be required for vesicle skin penetration. Both mixed micelles and 
standard liposome formulations of insulin failed to reduce blood glucose. It was thus concluded that Transfersomes provide non-invasive transdermal delivery of therapeutic agents, including insulin [64-66].

Successful topical delivery of low molecular weight heparin was reported after incorporation into flexible vesicles made of lipids with Tween 80 , which bore a surface charge. These vesicles were termed flexosomes of which cationic structures were the most efficient [67].

Topically applied Transfersomes have been evaluated for topical immunization using Gap junction proteins (GJP). The study revealed a rise in specific antibody levels marginally higher than those elicited by subcutaneous injections of GJP in Transfersomes, mixed lipid micelles or liposomes. The latter two carrier systems give no significant biological response after topical administration. Transdermal protein delivery by means of Transfersomes increased the relative concentration of anti-GJP IgA in the serum [68].

Transfersomes, niosomes (Span 85 and cholesterol, 1:1 w/w) and liposomes (PC and $\mathrm{CH}, 7: 3 \mathrm{w} / \mathrm{w}$ ) were compared for their relative potential in non-invasive transdermal delivery of tetanus toxoid (TT). The in vivo study revealed that topical TT-containing Transfersomes could elicit an immune response (anti-TT-IgG) equivalent to that produced following intramuscularly alum-adsorbed TT-based immunization whilst i.m. delivered niosomes and liposomes produced weaker immune responses [69].

A new type of fusogenic vesicles (vesosomes) have been introduced for topical immunization. In these, the tetanus toxoid was incorporated into cationic liposomes made of PC, dioleoyl phosphatidylethanolamine (DOPE) and dioleoyl trimethyl ammonium 
propane (DOTAP). These vesicles were subsequently entrapped in an interdigitated lipid bilayer to provide vesicles within a vesicle (vesosomes). These aggregates were claimed to provide a promising system for transcutaneous immunization [70].

Niosomes made of span 85 and cholesterol and prepared by a reversed phase evaporation technique were employed for topical delivery of a DNA vaccine. The DNA encoding hepatitis B surface antigen ( $\mathrm{HBsAg}$ ) was encapsulated in niosomes or liposomes. Topical application of niosomes produced a comparable serum antibody titer and endogenous cytokines levels when compared to intramuscular administration of recombinant HBsAg and topical liposomes. This was taken as an indication of the potential of niosomes as DNA vaccine carriers for effective topical immunization [71]. In a follow on study, cationic Transfersomes made of dioleoyl trimethyl ammonium propane (cationic lipid) with sodium deoxycholate were likewise successfully used for transdermal delivery of the hepatitis B DNA vaccine. Topical application of DNA loaded cationic Transfersomes elicited a comparable serum antibody titer and endogenous cytokines levels as produced after intramuscular recombinant HBsAg administration [72].The Span 80 based ultradeformable vesicles which was initially optimized by El Maghraby et al (2000a) [50] have also recently been used for topical immunization. The results suggested that hepatitis B loaded ultradeformable vesicles are able to provide protective immune response [73-74].

The efficiency of Hepatitis B surface antigen (HBsAg)-loaded ethosomes was evaluated for transcutaneous immunization against Hepatitis B. Transcutaneous delivery potential of the antigen-loaded system using human cadaver skin demonstrated a much higher skin permeation of the antigen in comparison to conventional liposomes and 
soluble antigen preparations. Topically applied HBsAg-loaded ethosomes to mice showed a robust systemic and mucosal humoral immune response compared to intramuscularly administered alum adsorbed HBsAg suspension, topically applied plain HBsAg solution and hydroalcoholic solution of HBsAg. It was thus concluded that HBsAg-loaded ethosomes can generate a protective immune response and their ability to traverse and target the immunological milieu of the skin may find application in the development of a transcutaneous vaccine against the Hepatitis B virus (HBV) [75].

\subsection{Mechanisms of action of vesicles as skin drug delivery systems}

Various mechanisms have been reported for improved transdermal drug delivery from vesicular systems. These mechanisms are summarized in Figure 3. This section will critically evaluate the reported mechanisms by highlighting both positive and negative evidence for each mechanism.

\section{Insert Figure 3}

\subsubsection{Free drug mechanism (see Figure 3 at A)}

According to this process, the drug has to be released from the vesicles before independent permeation into and through the skin [45]. In this case, vesicles can be considered only as carriers that can control drug release with drug permeation depending on its physicochemical chemical characteristics. To investigate this possibility, the transepidermal flux profile obtained from different liposome formulations was compared with the corresponding in vitro drug release profile. For all preparations, the peak flux of 
estradiol through skin appeared at a time during which drug release was negligible. This suggests that a free drug mechanism did not operate for the tested standard and deformable liposome formulations [49].

\subsubsection{Penetration enhancing mechanism (see Figure 3 at B)}

According to this mechanism, vesicle components may enter the skin as monomers disrupting the packing characteristics of the SC lipid bilayers and thus enhancing drug permeation. The penetration enhancing effect of egg lecithin (included in a drug solution in propylene glycol) was first recorded two decades ago, after in vitro and in vivo animal studies. It was concluded that lecithin enhances the transdermal delivery of bunazosin hydrochloride by lowering the permeability barrier of the skin [76]. This early finding suggested the possible accelerant effect of a liposome component.

Freeze fracture electron microscopy and small angle X-ray scattering studies, performed 48 hours after incubating human SC in liposome dispersions, revealed that vesicle components can change the ultrastructure of the intercellular lipid regions, indicative of penetration enhancing effects. It was concluded that vesicles made of lipids with relatively small hydrophilic head groups can produce marked interaction with human stratum corneum lipids in vitro [77]. After application of soybean PC liposomes to human epidermis reconstituted in vitro, electron microscopy showed the presence of dose-dependent alterations in the morphology of both the SC and the viable epidermis with shrunken lipid droplets formed between the corneocytes. This supported further the previous findings of an enhancing action from liposomes [78].

In another study, differential scanning calorimetric investigations performed to human SC treated (non-occlusively) with dimyristoylphosphatidylcholine (DMPC) 
liposomes showed changes in the enthalpy of the lipid-related transitions of the SC [79]. In addition, depending on composition, vesicles may produce an enhancing effect (shown by skin pre-treatment), may penetrate deep into the stratum corneum or may fuse and mix with skin lipid. Liposomes containing dioleylphosphatidylethanolamine (DOPE) or lysoPC produced the greatest effect [80]. Vesicles containing DOPE can fuse and mix with skin lipids and loosen their structure. This was evidenced by the interactions of these vesicles with stratum corneum lipid liposomes; it was suggested that the conical shape of DOPE was essential for this effect. Both the PE and dioleyl moieties were essential, as nano-structures containing PE (with other fatty acid chains) provided lower enhancing effects compared with the DOPE liposomes [80]. More recently, the same group recorded deeper penetration of a lipophilic fluorescent probe into SC after application of PC liposomes containing 32\% ethanol compared with ethanol-free vesicles. Ethanol did not affect the penetration pattern from DOPE-containing liposomes, but addition of ethanol increased the mixing of both vesicles with SCLL. In addition, ethanol-containing nanostructures (both types) destabilized skin lipid-based liposomes as evidenced by increased calcein release from SCLL preparations compared with control (containing the same concentration of ethanol). These studies provide further evidence for the penetration enhancing effect of liposome components [81].

Phospholipid solutions in propylene glycol increased percutaneous absorption of indomethacin and the order of activity was; phosphatidylglycerol (PG) > phosphatidylethanolamine $(\mathrm{PE})>\mathrm{PC}>$ phosphatidylserine $(\mathrm{PS})>$ phosphatidic acid $(\mathrm{PA})$ $>$ phosphatidylinositol $(\mathrm{PI})>$ control > sphingomyelin $(\mathrm{SM})$ [82]. The phospholipids were classified into three groups; the first included PG, PE and PC with head groups 
having a strong enhancing effect, the second contained PS, PA and PI, classified as weak promoters and the third has SM which is not an enhancer. When repeating the same study on silastic membranes instead of skin, no phospholipid accelerated the permeation of the drug. Furthermore, PG, PE, PC or SM did not significantly affect the percutaneous absorption of indomethacin through skin lacking stratum corneum. This clearly indicated that phospholipids act directly on the permeability barrier of SC [82]. In a similar study, the effects of the hydrophobic entity in the phospholipids were probed [83]. It was suggested that phospholipids containing unsaturated fatty acid chains in the hydrophobic group are strong promoters. As in the previous studies, the authors showed that the phospholipids work by directly affecting the SC.

The effect of skin pretreatment with PC liposomes on the transdermal delivery of a variety of corticosteroids from creams was evaluated by the human skin blanching assay. The pre-treatment increased the blanching response and reduced the tachyphylaxis for all preparations except clobetasone butyrate. The authors explained this effect on the basis that PC may form a thin film on the skin surface into which corticosteroids can preferentially partition, or that $\mathrm{PC}$ can partition into SC and thus enhance delivery by influencing the partitioning of corticosteroid into skin [84].

Negative findings regarding the penetration enhancing mechanism have also been reported, some examples of which are summarized below. No accelerant effect was found for SCLL after application of the empty vesicles in combination with free interferon [57]. Skin pretreatment with empty phospholipids or skin lipid liposomes did not give the advantages of encapsulated drug, showing no enhancement for both hydrophilic and lipophilic drugs [85]. It was thus concluded that the suggestion that 
liposomes interact with SC is invalid. It was further proposed that vesicles must at least be applied concomitantly with the drug or that the drug must be encapsulated within them.

Employing a pre-treatment protocol with empty vesicles, we evaluated the penetration enhancing effect of a variety of vesicles [49], including both ultradeformable and standard formulations. The ultradeformable (UD) systems included PC with sodium cholate (UD1), PC with Span 80 (UD2) and PC with Tween 80 (UD3) which were previously optimized. The standard liposomes (SL) encompassed pure PC vesicles (non rigid, SL1), PC with CH (membrane stabilized, SL2), and two rigid liposomes of DPPC (SL3) and DPPC/CH (SL4). The results indicated a possible accelerant effect only for the non-rigid PC vesicles (SL1) for which drug flux improved 4-fold. However, comparing this promotion with the relative flux obtained after application of estradiol encapsulated in the same formulation (8-fold) it was concluded that the penetration enhancing mechanism is not the only (or main) mechanism operating. For other traditional and ultradeformable systems, skin pre-treatment was not effective, thus excluding an accelerant effect and suggesting other mechanisms for the improved skin delivery from such formulations.

The importance of vesicular structure for transdermal delivery from liposomes was investigated by comparing estradiol skin delivery from standard and ultradeformable vesicles with that obtained from propylene glycol solutions containing the same vesicular components. All systems were at the same thermodynamic activity. The results indicated the importance of the colloidal structure and these findings again excluded a major role for a penetration promoting mechanism in improved skin delivery from such liposomes 
[86]. More recently, the efficiency of elastic and rigid niosomes was investigated and it was reported that drug molecules must be applied together with, and entrapped within, the nano-aggregates themselves. It was suggested that elastic vesicles act as drug carrier systems and not as penetration enhancers [55].

\subsubsection{Vesicle adsorption to and/or fusion with the stratum corneum (see Figure 3 at C)}

The processes of adhesion onto the skin surface and fusion or mixing with the lipid matrix of stratum corneum have been suggested for liposome lipids [80]. The phospholipid components of liposomes can rapidly enter the skin with the drugs following their fate [87]. Phospholipids increased the partitioning of estradiol, progesterone and propranolol into the stratum corneum lipid bilayers [88]. It was also reported that the phospholipid component of liposomes can increase the continuity of the lipid matrix of the skin thus facilitating the movement of lipophilic molecules [89]. Based on this suggestion, we should expect improved drug uptake from a saturated aqueous solution after skin pre-treatment with empty vesicles. Consequently, an uptake study was conducted in which stratum corneum membranes were dipped for a short time (10 minutes) into medicated vesicles or into an aqueous drug solution with or without pre-treatment with empty vesicles [49]. Drug uptake increased only from medicated carriers and the uptake ratios (UR) between the vesicles and solution ranged from 23 to 29 with no significant differences between ultradeformable and standard liposomes. Correlating the superiority of ultradeformable vesicles over standard liposomes in increasing transepidermal flux, with no significant difference found in the UR at short 
contact time, suggests that deformable vesicles either improved drug diffusion or penetrated deeper in the epidermis, thus allowing more efficient drug clearance

\subsubsection{Intact vesicular skin penetration mechanism (see Figure 3 at D)}

\subsubsection{Standard liposomes}

The hypothesis that intact vesicles penetrate through human skin was suggested in the first report on liposomes as skin drug delivery systems [12, 19]. It was difficult to believe that large lipid vesicles could penetrate the densely packed stratum corneum in great numbers. Accordingly, many researchers have tested this concept with controversial findings being reported. Electron micrography showed the presence of intact liposomes in the dermis after application of liposomes loaded with an electron dense marker to guinea pigs. The authors proposed that liposomes can penetrate the epidermis carrying the drug [27].

Liposomal formulations were superior in the treatment of eczema but not for psoriasis compared to a traditional gel [90]. This finding suggested that vesicles can penetrate diseased skin with its ruptured SC (as in eczema) but cannot invade skin with hyperkeratosis, as in psoriasis. Subsequently, fluoromicrographic studies showed that intact small unilamellar vesicles (SUVs) containing $\mathrm{PC}$ and $\mathrm{CH}$ penetrated no deeper than the stratum corneum [91].

The ratio of radiolabelled components of liposomal preparations was constant throughout the skin strata after topical application of liposomes with dual labelled components; the finding were explained as possible molecular mixing of liposomal bilayers with the SC bilayers [59]. The ratio of the radiolabelled marker to liposome components was also constant throughout skin strata. The explanation given by the 
authors (molecular mixing) may not justify equal ratios of the dual label in the deeper skin strata. These findings may suggest possible carrier skin penetration. Similar findings were reported again for both phospholipid and stratum corneum lipid-based liposomes [47].

In contrast to the above findings, intermediate-sized and not the small liposomes resulted in higher skin deposition, indicating that intact liposomes did not penetrate the skin [92]. In addition, no evidence of intact carrier penetration could be found after application of DMPC or soy-lecithin liposomes [78-79].

The ratio of $\left[{ }^{3} \mathrm{H}\right]$ DPPC to $\left[{ }^{14} \mathrm{C}\right]$ tretinoin deposited into various skin strata of the hairless rat was monitored after application of liposome-encapsulated tretinoin. This ratio was constant throughout the $\mathrm{SC}$ but not in the nucleated epidermis and dermis. Liposomes and tretinoin co-transport into SC was thus accompanied by independent penetration of free drug, which could have escaped from liposomes on the skin surface [93]. This report suggests that vesicles can penetrate only into the stratum corneum.

\subsubsection{Ultradeformable and elastic vesicles}

Transfersomes have been reported to penetrate the skin intact and go deep enough to reach the systemic circulation. The driving force for Transfersome skin transfer is thought to be the natural transdermal hydration gradient. Phospholipid hydrophilicity leads to xerophobia (tendency to avoid dry surrounding). Accordingly, for the vesicles to remain maximally swollen, those on the skin surface will try to follow the local hydration gradient, moving into deeper skin strata $[13,52,65]$.

The process of Transfersome skin penetration was attributed to the vesicle high deformability (Figure 4) which is imparted by the presence of edge activator molecules 
(e.g surfactants) which tend to accumulate at the site of high stress due to their raised propensity for greatly curved structures. This rearrangement reduces the energy required for deformation. The stress is thought to be produced upon drying of the vesicles which, being flexible, can follow the transdermal hydration gradient [65].

\section{Insert Figure 4}

To test this hypothesis, the skin delivery of estradiol from large multi-lamellar vesicles (LMLVs, at least $557 \mathrm{~nm}$ in diameter) was compared with that obtained from smaller vesicles of a mean size of $124-138 \mathrm{~nm}$, (SUVs), assuming that penetration is a function of the vesicle size [49]. The tested SUVs were less than the maximum dimension reported to enter skin and the minimum size of tested LMLVs was above the maximum volume which can invade skin [65]. The SUVs were as effective as LMLVs, a finding which suggests that intact vesicles do not permeate through human epidermal membrane in vitro [49]. However, it should be noted that the LMLVs were prepared by mechanical shaking, a technique which produces heterogeneous populations of vesicles (i.e. a mix of smaller and larger sized structures). In more recent studies, reducing vesicle size improved drug deposition into deeper strata and penetration through skin, with large structures improving deposition only [94-95].

Dipotassium glycyrrhizinate (DG), an amphiphilic anti-inflammatory drug, was incorporated into liposomes [96]; DG was reported to increase the elasticity of the carrier. Measuring the vesicle size before and after extrusion through a microporous filter, the vesicles were capable of penetrating a pore with a diameter three times smaller than their 
own span. This was taken as evidence for elasticity but the liposomes only improved skin deposition of DG, not flux. This finding provides evidence that vesicles can penetrate into but not through skin. In similar studies, the size of Transfersomes did not change after extrusion through semi-permeable membrane barriers. The authors reported the presence of the vesicles in mouse blood after topical application of fluorescent labeled Transfersomes. Surprisingly, the size of these vesicles was similar to that of the starting liposome suspension. This was taken evidence for intact vesicle invasion into and through skin [97-98].

Fast delivery of intact elastic niosomes into the SC was recorded and was thought to be via channel-like regions in the SC. Again, elastic vesicles were superior to rigid ones with non-occlusive application being optimum [99]. Monitoring the co-transport of vesicle components and a model drug into human skin in vivo, it was found that only elastic vesicle materials can rapidly enter the SC reaching almost the SC-viable epidermal junction. However, the distribution profile of the drug in the lower SC layers was different from that of the vesicle components. This suggests that drug release can take place once the elastic vesicles partition into the SC [56].

Based on the above reports, it appears that some vesicles may penetrate intact to some extent into healthy skin. However what is not clear is how deep into the skin strata intact carriers move, and if indeed intact vesicles can carry their contents through the entire tissue and reach the blood stream.

\subsubsection{Trans-appendageal penetration (see Fig. 3 at E)}


Open application was recommended for optimal transdermal drug delivery from Transfersomes, with occlusive application and full skin hydration being detrimental. This was attributed to inhibition of the transdermal hydration gradient, which is believed to be the driving force for Transfersome-skin penetration [65]. Another possible explanation is that occlusion and excessive hydration of the skin can swell the corneocytes and thus close or at least minimize the size of shunts that may play a role in liposomal skin delivery.

Electron microscopy indicated that liposomes up to $600 \mathrm{~nm}$ diameter can penetrate through skin but those of $1000 \mathrm{~nm}$ or more remain interiorised in the SC. Deposition depended on the hair density of the skin with higher deposition recorded in hairy guinea pigs. However, with regard to penetration though skin, no difference could be found between hairless and hairy guinea pigs. Despite this, it was concluded that invasion is mainly along the hair sheath [100]. However, these findings only reflect delivery into, rather than through, the hair follicles. Other workers excluded vesicular delivery through shunts after recording no significant variations between different animals or humans with diverse densities of hair follicles, with regard to the Transfersomal input of insulin [66].

A novel in vitro technique using human abdominal skin was developed to explore the role of appendageal transport on liposomal skin delivery of estradiol. The proponents of this technique utilized a sandwich of SC overlying an entire epidermal membrane (including its stratum corneum) and monitored vesicular drug delivery through this sandwich compared with the delivery through the epidermis only. In this system, the shunt orifices occupy only a small fraction of the total skin surface area, and thus there is 
negligible opportunity for the shunts in the two membranes (in the skin sandwich) to superimpose. It was thus assumed that the top layer of SC would block most of the shunts available in the bottom membrane [101]. From this study, it was concluded that the shunt routes played a minor role in estradiol transdermal delivery from liposomes. Another group of researchers recorded enhanced transfollicular delivery from liposomes only after combination with iontophoresis [102].

From the above literature, it appears that the shunt routes play no major role in liposomal transdermal drug delivery. However, vesicle penetration into but not necessarily through hair follicles (i.e. targeting) is clearly demonstrated by numerous literature reports.

\section{Conclusion}

The potential of different vesicles for dermal and transdermal drug delivery is now evident. Recent modifications and structural and composition optimization have generated improved therapeutic potential. These modifications resulted in the development of flexible and ultradeformable liposomes for which great claims have been made with respect of enhanced transdermal drug delivery, including efficiencies comparable with subcutaneous administration. Indeed, some claims include noninvasive transcutaneous vaccination but the mechanisms of action of these vesicles as skin drug delivery systems require further clarification.

\section{Expert opinion}

The use of vesicles as skin drug delivery systems was proposed almost three decades ago with the goal of localizing a corticosteroid in the skin strata, thus reducing its systemic side effects. These initial studies utilized standard liposomes made of 
phospholipids with or without cholesterol. Such liposomes encapsulated mainly small organic compounds and rarely macromolecules such as interferon. The recorded effects were mainly improved skin deposition and rarely enhanced transdermal delivery. Subsequently, considerable interest was directed towards vesicular transdermal drug delivery through the introduction of Transfersomes. These were developed by incorporating edge activators (surfactants) into the vesicular components to produce ultradeformable vesicles. These were claimed to deliver both small and large molecular weight compounds including insulin into and through the skin with efficiency similar to subcutaneous administration. However, the mechanism of action of these carriers remains controversial, especially the claim that intact ultradeformable vesicles can penetrate the skin deep enough to reach the systemic circulation with some vesicles detected in the blood after topical administration. In parallel to Transfersomes, niosomes were developed with the vesicles made of surfactants with cholesterol. These niosomes also showed some success in dermal and transdermal delivery and again flexible niosomes appear superior to more rigid structures. The elasticity of the vesicular membrane was also achieved by incorporating ethanol in the vesicular membrane, producing ethosomes. These ultradeformable and elastic vesicles are also claimed to be valuable for non-invasive transdermal vaccination. Taken together, the literature and our own research indicates strongly that some types of liposomes can deliver both small and macromolecular therapeutic agents to the skin, that delivery through the skin may possibly be increased but that some literature claims appear to be rather extravagant. 
Despite numerous encouraging reports on flexible and ultradeformable vesicles, we think that high vesicle flexibility and deformability is always associated with reduced stability, particularly manifested as loss of entrapped material. Not only is this intuitive, we have also modeled the geometry of a deformable vesicle as it reconfigures to enter the narrow SC lipid domain; on doing so the volume within the liposome cannot be conserved so contents must be expelled. This problem may be partially responsible for the lack of deformable vesicular transdermal drug delivery systems in the market. Additionally, stability of vesicular systems is problematic, again with respect to drug retention during storage. This is especially problematic for flexible / deformable (i.e. fluid) liposomes. One approach to overcome this instability is to minimize the water content of the formulation by developing a vesicle pro-concentrate, so called proliposomes or proniosomes which contain all the vesicular components with ethanol and only traces of water. These systems are gel like preparations which are believed to generate vesicles upon application to skin after dilution with skin secretions before delivering their contents into and through the skin. However, the application of this approach requires further investigation to test the behavior of the vesicles after in situ formation.

A further issue affecting vesicular system commercialization is problems in scaling up, further complicated when considering that the majority of reports utilized simple vesicular dispersion with very few authors preparing pharmaceutical formulations (e.g. gels or patches). Accordingly, in addition to developing stable liposomes, some understanding of realistic formulation design is necessary. Questions remain about whether vesicular incorporation into the pharmaceutical dosage form will affect their 
topical and transdermal drug delivery capacity. This issue will depend on the mechanism of action of elastic and deformable vesicle. Despite numerous reports and controversy over mechanisms of action, it is widely accepted that intact deformable and flexible vesicles can enter human skin to some depth. The next 5 years will show whether small and macromolecular delivery via the skin using vesicles is a commercial reality. 


\section{References}

1- Barry BW. Dermatological Formulations: Percutaneous Absorption, Marcel Dekker, New York and Basel, 1983.

2- Barry BW. Novel mechanisms and devices to enable successful transdermal drug delivery. Eur J Pharm Sci 2001; 14: 101-114.

3- Williams AC. Transdermal and Topical Drug Delivery; from theory to clinical practice. Pharmaceutical Press, London, 2003.

4- Williams AC, Barry BW. Penetration enhancers. Adv Drug Del Rev 2004; 56: 603-618.

5- Megrab NA, Williams AC, Barry BW. Oestradiol permeation across human skin and silastic membranes: effects of propylene glycol and supersaturation. J Control Rel. 1995; 36: 277-294.

6- Leichtnam ML, Rolland H, Wüthrich P, Guy RH. Impact of antinucleants on transdermal delivery of testosterone from a spray. J Pharm Sci 2007; 96: 84-92.

7- Miller LL, Kolaskie CJ, Smith GA, Rivier J. Transdermal iontophoresis of gonadotropin releasing hormone and two analogues. J Pharm Sci 1990; 79: 490493.

8- Prausnitz MR, Gimm JA, Guy RH, Langer R, Weaver JC, Cullander C. Imaging of transport pathways across human stratum corneum during high-voltage and low-voltage electrical exposures. J Pharm Sci 1996; 85: 1363-1370.

9- Kost J, Pliquett U, Mitragotri S, Yamamoto A, Langer R, Weaver J. Synergistic effect of electric field and ultrasound on transdermal transport. Pharm Res 1996; 13: $633-638$. 
10- Kreilgaard M. Influence of microemulsions on cutaneous drug delivery, Advanced Drug Deliv Rev 2002; 54: S77-S98.

11-El Maghraby GM. Transdermal delivery of hydrocortisone from eucalyptus oil microemulsion: Effects of cosurfactants. Int J Pharm 2008; 355: 285-292.

12- Mezei M, Gulasekharam V. Liposomes-a selective drug delivery system for topical route of administration. 1. lotion dosage form. Life Sci 1980; 26: 14731477.

13-Cevc G, Blume G. Lipid vesicles penetrate into intact skin owing to the transdermal osmotic gradients and hydration force. Biochim Biophys Acta 1992; 1104: 226-232.

14- Schreier H, Bouwstra J. Liposomes and niosomes as topical drug carriers: dermal and transdermal drug delivery. J Control Rel 1994; 30: 1-15.

15-El Maghraby GM, Williams AC, Barry BW. Can drug bearing liposomes penetrate intact skin? J Pharm Pharmacol 2006; 58: 415-429.

16- Touitou E, Godin B, Weiss C. Enhanced delivery of drugs into and across the skin by ethosomal carriers. Drug Dev Res 2000; 50: 406-415.

17- Touitou E, Dayan N, Bergelson L, Godin B, Eliaz M. Ethosomes - novel vesicular carriers for enhanced delivery: characterisation and skin penetration properties. J Control Rel 2000; 65: 403-418.

18- El Maghraby GM, Williams AC, Barry BW. Liposomes and skin. From drug delivery to model membranes. Eur J Pharm Sci 2008; 34: 203-222. 
19- Mezei M, Gulasekharam V. Liposomes-a selective drug delivery system for topical route of administration: gel dosage form. J Pharm Pharmacol 1982; 34: 473-474.

20- Mezei M. Liposomes as a skin drug delivery system. In Breimer DD and Speiser P (eds.), Topics in Pharmaceutical Sciences, Elsevier, Amsterdam, New York, Oxford, 1985; pp. 345-358.

21-Vermorken AJM, Hukkelhoven MWAC, Vermeesch-Marksiag AMG, Goos CMAA, Wirtz P, Ziegenmeyer J. The use of liposomes in topical application of steroids. J Pharm Pharmacol 1984; 36: 334-336.

22- Wohlrab W, Lasch J, Laub R, Taube CM, Wellner K. Distribution of liposomeencapsulated ingredients in human skin ex vivo. In Falco OB, Korting HC, Maibach HI (eds), Liposome Dermatics, Springer-Verlag, Berlin 1992; pp. 217225.

23-Fresta M, Puglisi G. Corticosteroid dermal delivery with skin-lipid liposomes. J Control Rel 1997; 44: 141-151.

24- Yu HY, Liao HM. Triamcinolone permeation from different liposome formulations through rat skin in vitro. Int J Pharm 1996; 127: 1-7.

25-Erdogan M, Wright JR, McAlister VC. Liposomal tacrolimus lotion as a novel topical agent for immune-mediated skin disorders: experimental studies in a murine model. Br J Dermatol 2002; 146: 964-967.

26-Gesztes A, Mezei M. Topical anesthesia of skin by liposome-encapsulated tetracaine. Aneth Analg 1988; 67: 1079-1081. 
27-Foldvari M, Gesztes A, Mezei M. Dermal drug delivery by liposome encapsulation: Clinical and electron microscopic studies. J Microencaps 1990; 7(4): 479-489.

28- Foldvari M. In vitro cutaneous and percutaneous delivery and in vivo effecacy of tetracaine from liposomal and conventional vehicles. Pharm Res 1994; 11(11): 1593-1598.

29- Hung OR, Comeau L, Riley MR, Tan S, Whynot S, Mezei M. Comparative topical anaesthesia of EMLA and liposome-encapsulated tetracaine. Can. J Anaesth 1997; 44: 707-711.

30- Fisher R, Hung O, Mezei M, Stewart R. Topical anaesthesia of intact skin: Liposome-encapsulated tetracaine vs EMLA. Br J Anaesth 1998; 81: 972-973.

31- Planas ME, Gonzalez P, Rodriguez L, Sanchez S, Cevc G. Noninvasive percutaneous induction of topical analgesia by a new type of drug carrier, and prolongation of local pain insensitivity by anesthetic liposomes. Aneth Analg 1992; 75: 615-621.

32-El Maghraby GM, Williams AC, Barry BW. Skin delivery of 5-fluorouracil from ultradeformable and standard liposomes in vitro. J Pharm Pharmacol 2001; 53: 1069-1077.

33-Cevc G, Blume G, Schätzlein A. Transfersomes-mediated transepidermal delivery improves the regio-specificity and biological activity of corticosteroids in vivo. $\mathbf{J}$ Control Rel 1997; 45: 211-226. 
34- Manosroi A, Jantrawut P, Manosroi J. Anti-inflammatory activity of gel containing novel elastic niosomes entrapped with diclofenac diethylammonium Int J Pharm 2008; 360: 156-163.

35- Lauer AC, Ramachandran C, Leib LM, Niemiec S, Weiner ND. Targeted delivery to pilosebaceous unit via liposomes. Advanced Drug Del Rev 1996; 18: 311-324.

36- Lauer AC. Percutaneous drug delivery to the hair follicle. In: Bronaugh RL, Maibach HI (eds.), Percutaneous Absorption Drugs-Cosmetics-MechanismsMethodology, $3^{\text {rd }}$ Edition, Marcel Dekker, Inc., New York. Basel, 1999; pp. 427449.

37-Lieb LM, Ramachandran C, Egbaria K, Weiner N. Liposomally encapsulated active ingredients penetrate through the hair follicle. In Falco O B, Korting HC, Maibach HI (eds), Liposome Dermatics, Springer-Verlag, Berlin, 1992; pp. 200205.

38- Lieb LM, Ramachandran C, Egbaria K, Weiner N. Topical delivery enhancement with multilamellar liposomes into pliosebaceous units: I. In vitro evaluation using fluorescent techniques with hamster ear model. J Invest Dermatol 1992; 99(1): 108-113.

39- Lieb LM, Flynn G, Weiner N. Follicular (pilosebaceous unit) deposition and pharmacological behavior of cimetidine as a function of formulation. Pharm Res 1994; 11(10): 1419-1423.

40- Touitou E, Schaffer FL, Dayan N, Alhaique F, Riccieri F. Modulation of caffeine delivery by carrier design: liposomes versus permeation enhancers. Int J Pharm 1994; 103: 131-136. 
41-Tschan T, Steffen H, Supersaxo A. Sebaceous-gland deposition of isotretinoin after topical application: an in vitro study using human facial skin. Skin Pharmacol 1997; 10: 126-134.

42-Skalko N, Cajkovac M, Jalsenjak I. Liposomes with clindamycin hydrochloride in therapy of acne vulgaris. Int J Pharm 1992; 85: 97-101.

43- Meybeck A. Comedolytic activity of liposomal antiacne drug in an experimental model. In Falco OB, Korting HC, Maibach HI (eds), Liposome Dermatics, Springer-Verlag, Berlin, 1992; pp. 235-241.

44- Tabbakhian M, Tavakoli N, Jaafari MR, Daneshamouz S. Enhancement of follicular delivery of finasteride by liposomes and niosomes. 1. In vitro permeation and in vivo deposition studies using hamster flank and ear models. Int J Pharm 2006; 323: 1-10.

45-Ganesan MG, Weiner ND, Flynn GL, Ho NFH. Influence of liposomal drug entrapment on percutaneous absorption. Int J Pharm 1984; 20: 139-154.

46- Bonina FB, Montenegro L, Scrofani N, Esposito E, Cortesi R, Menegatti E, Nastruzzi C. Effects of phospholipid based formulations on in vitro and in vivo percutaneous absorption of methyl nicotinate. J Control Rel 1995; 34: 53-63.

47- Fresta M, Puglisi G. Application of liposomes as potential cutaneous drug delivery systems. In vitro and in vivo investigation with radioactivity labelled vesicles. J Drug Target 1996; 4: 95-101.

48- Simoes SI, Martins MBF, Cruz MEM, Cevc G. Anti-inflammatory effects of $\mathrm{Cu}$, Zn-superoxide dismutase in liposomes, Transfersomes or micelles, in the acute murine ear edema model. In Brain KR, Walters KA (eds), Perspectives in 
Percutaneous Penetration, sixth International Conference, Vol. 6a, STS Publishing, Cardiff, 1998; p. 50.

49-El Maghraby GM, Williams AC, Barry BW. Skin delivery of oestradiol from deformable and traditional liposomes: Mechanistic studies. J Pharm Pharmacol 1999; 51: 1123-1134.

50-El Maghraby GM, Williams AC, Barry BW. Oestradiol skin delivery from ultradeformable liposomes: refinement of surfactant concentration. Int J Pharm 2000; 196: 63-74.

51-El Maghraby GM, Williams AC, Barry BW. Interactions of surfactants (edge activators) and skin penetration enhancers with liposomes. Int J Pharm 2004; 276 : $143-161$.

52-Cevc G. Rational for the production and dermal application of lipid vesicles. In Falco OB, Korting HC, Maibach HI (eds), Liposome Dermatics, Springer-Verlag, Berlin, 1992; pp. 82-90.

53-Cevc G. Material transport across the permeability barriers by means of lipid vesicles. In Lipowsky R, Sackmann E (eds), Handbook of Biological Physics, Vol. 1, Elsevier Science B.V., North Holland, Amsterdam, 1995; pp. 465-490.

54-Cevc G. Transfersomes, liposomes and other lipid suspensions on the skin: permeation enhancement, vesicle penetration, and transdermal drug delivery. Crit Rev Ther Drug Car Sys 1996; 13(3\&4): 257-388.

55- Honeywell-Nguyen PL, Arenja S, Bouwstra JA. Skin penetration and mechanisms of action in the delivery of the D2-agonist rotigotine from surfactant-based elastic vesicle formulations. Pharm Res 2003; 20(10):1619-1625. 
56- Honeywell-Nguyen PL, Gooris GS, Bouwstra JA. Quantitative assessment of the transport of elastic and rigid vesicle components and a model drug from these vesicle formulations into human skin in vivo. J Invest Dermatol 2004; 123(5): 902-910.

57-Weiner N, Williams N, Birch G, Ramachandran C, Shipman CJR, Flynn G. Topical delivery of liposomally encapsulated interferon evaluated in cutaneous herpes guinea pig model. Antimicrob Agents Chemother 1989; 33(8): 1217-1221.

58-Egbaria K, Ramachandran C, Kittayanond D, Weiner N. Topical delivery of liposomally encapsulated interferon evaluated by in vitro diffusion studies. Antimicrob Agents Chemother 1990; 34(1): 107-110.

59-Egbaria K, Ramachandran C, Weiner N. Topical delivery of cyclosporin: evaluation of various formulations using in vitro diffusion studies in hairless mouse skin. Skin Pharmacol 1990; 3: 21-28.

60- Du Plessis J, Egbaria K, Ramachandran C, Weiner ND. Topical delivery of liposomally encapsulated gamma-interferon. Antiviral Res 1992; 18: 259-265.

61- Artman C, Roding J, Ghyczy M, Prazel HG. Liposomes from soya phospholipids as percutaneous drug carriers: Qualitative in vivo investigations with antibodyloaded liposomes. Arzneium Forsch Drug Res 1990; 40(II): 1363-1365.

62- Artman C, Roding J, Ghyczy M, Prazel HG. Liposomes from soya phospholipids as percutaneous drug carriers: Quantitative in vivo investigations with radioactivity labelled liposomes. Arzneium. Forsch Drug Res 1990; 40(II): 13651368. 
63- Short SM, Paasch BD, Turner JH, Weiner N, Daugherty AL, Mrsny RJ. Percutaneous absorption of biologically-active interferon-gamma in a human skin graft-nude mouse model. Pharm Res 1996; 13(7): 1020-1027.

64-Cevc G, Schätzlein A, Gebauer D, Blume G. Ultra-high efficiency of drug and peptide transfer through the intact skin by means of novel drug-carriers, transfersomes. In Brain KR, James VA, Walters KA (eds), Prediction of Percutaneous Penetration, $3^{\text {rd }}$ International Conference, 14-16 April, Vol. 3b, STS Publishing, Cardiff, 1993; pp. 226-236.

65-Cevc G, Schätzlein A, Blume G. Transdermal drug carriers: basic properties, optimization and transfer efficiency in the case of epicutaneously applied peptides. J Control Rel 1995; 36: 3-16.

66-Cevc G, Gebauer D, Stieber J, Schätzlein A, Blume G. Ultraflexible vesicles, Transfersomes, have an extremely low pore penetration resistance and transport therapeutic amounts of insulin across the intact mammalian skin. Biochim Biophys Acta 1998; 1368: 201-215.

67-Song YK, Kim CK. Topical delivery of low-molecular-weight heparinwith surface-charged flexible liposomes. Biomaterials 2006; 27: 271-280.

68- Paul A, Cevc G, Bachhawat BK. Transdermal immunisation with an integral membrane component, gap junction protein, by means of ultradeformable drug carriers, Transfersomes. Vaccine 1998; 16: 188-195.

69- Gupta PN, Mishra V, Rawat A, Dubey P, Mahor S, Jain S, Chatterji DP, Vyas SP. Non-invasive vaccine delivery in transfersomes, niosomes and liposomes: a comparative study. Int J Pharm 2005; 293: 73-82. 
70-Mishra V, Mahor S, Rawat A, Dubey P, Gupta PN, Singh P, Vyas SP. Development of novel fusogenic vesosomes for transcutaneous immunization. Vaccine 2006; 24: 5559-5570.

71- Vyas SP, Singh RP, Jain S, Mishra V, Mahor S, Singh P, Gupta PN, Rawat A, Dubey P. Non-ionic surfactant based vesicles (niosomes) for non-invasive topical genetic immunization against hepatitis B. Int J Pharm 2005; 296: 80-86.

72-Mahor S, Rawat A, Dubey PK, Gupta PN, Khatri K, Goyal AK, Vyas SP. Cationic transfersomes based topical genetic vaccine against hepatitis B. Int $\mathbf{J}$ Pharm 2007; 340: 13-19.

73- Mishra D, Dubey V, Asthana A, Saraf DK, Jain NK. Elastic liposomes mediated transcutaneous immunization against Hepatitis B. Vaccine 2006; 24: 4847-4855.

74- Mishra D, Mishra PK, Dubey V, Dabadghao S, Jain NK. Evaluation of uptake and generation of immune response by murine dendritic cells pulsed with hepatitis B surface antigen-loaded elastic liposomes. Vaccine, 2007; 25(39-40):6939-6944.

75- Mishra D, Mishra PK, Dubey V, Nahar N, Dabadghao S, Jain NK. Systemic and mucosal immune response induced by transcutaneous immunization using Hepatitis B surface antigen-loaded modified liposomes. Eur J Pharm Sci 2008; 33: 424-433.

76- Kato A, Ishibashi Y, Miyake Y. Effect of egg yolk lecithin on transdermal delivery of bunazosin hydrochloride. J Pharm Pharmacol 1987; 39: 399-400.

77- Hofland HEJ, Bouwstra JA, Bodde HE, Spies F, Junginger HE. Interaction between liposomes and human stratum corneum in vitro: freeze fracture electron 
microscopical visualization and small angle X-ray scattering studies. $\mathrm{Br} \mathrm{J}$ Dermatol 1995; 132: 853-866.

78- Korting HC, Stolz W, Schmid MH, Maierhofer G. Interaction of liposomes with human epidermis reconstructed in vitro. Britsh J Dermatol 1995; 132: 571-579.

79-Zellmer S, Pfeil W, Lasch J. Interaction of phosphatidylcholine liposomes with the human stratum corneum. Biochim Biophys Acta 1995; 1237: 176-182.

80- Kirjavainen M, Urtti A, Jaaskelainen I, Suhonen TM, Paronen P, Koskela RV, Kiesvaara J, Monkkonen J. Interaction of liposomes with human skin in vitro - the influence of lipid composition and structure. Biochim Biophys Acta 1996; 1304: 179-189.

81- Kirjavainen M, Urtti A, Koskela RV, Kiesvaara J, Monkkonen J. Liposome-skin interactions and their effects on the skin permeation of drugs. Eur J Pharm Sci 1999; 7: 279-286.

82- Yokomizo Y, Sagitani H. Effects of phospholipids on the percutaneous penetration of indomethacin through the dorsal skin of the guinea pig, in vitro. $\mathrm{J}$ Control Rel 1996; 38: 267-274.

83- Yokomizo Y, Sagitani H. Effects of phospholipids on the percutaneous penetration of indomethacin through the dorsal skin of the guinea pig, in vitro. 2 . The effects of hydrophobic group in the phosphlipids and a comparison with general enhancers. J Control Rel 1996; 42: 37-46.

84- Jacobs M, Martin GP, Marriott C. Effects of phosphatidylcholine on the topical bioavailability of corticosteroids assessed by the human skin blanching assay. $\mathbf{J}$ Pharm Pharmacol 1988; 40: 829-833. 
85- Du Plessis J, Weiner N, Muller DG. The influence of in vivo treatment of skin with liposomes on the topical absorption of a hydrophilic and a hydrophobic drug in vitro. Int J Pharm 1994; 103: $\mathrm{R}_{1}-\mathrm{R}_{5}$.

86- El Maghraby GM, Williams AC, Barry BW. Skin delivery of oestradiol from lipid vesicles: importance of liposome structure. Int J Pharm 2000; 204: 159-169.

87-Kim MK, Chung SJ, Lee MH, Cho AR, Shim CK. Targeted and sustained delivery of hydrocortisone to normal and stratum corneum-removed skin without enhanced skin absorption using a liposome gel. J Control Rel 1997; 46: 243-251.

88- Kirjavainen M, Monkkonen J, Saukkosaari M, Koskela RV, Kiesvaara J, Urtti A. Phospholipids affect stratum corneum lipid bilayer fluidity and drug partitioning into the bilayers. J Control Rel 1999; 58: 207-214.

89- Keith AD, Snipes W. Phospholipids as moisturizing agents. In Frost P. Horovitz S (eds.), Principles of Cosmetics for the Dermatologist, The C.V. Mosby Company: St. Louis, 1982; pp 59-69.

90- Korting HC, Zeinicke H, Schafer KM, Falco OB. Liposome encapsulation improves efficacy of betamethasone dipropionate in atopic eczema but not in psoriasis vulgaris. Eur J Clin Pharmacol 1990; 39: 349-351.

91- Lasch J, Laub R, Wohlrab W. How deep do intact liposomes penetrate into human skin? J Control Rel 1991; 18: 55-58.

92- Du Plessis J, Ramachandran C, Weiner N, Muller DG. The influence of particle size of liposomes on the disposition of drug into skin. Int J Pharm 1994; 103: 277282. 
93- Masini V, Bonte F, Meybeck A, Wepierre J. Cutaneous bioavailability in hairless rats of tretinoin liposomes or gel. J Pharm Sci 1993; 82(1): 17-21.

94- Valenta C, Janisch M. Permeation of cyproterone acetate through pig skin from different vehicles with phospholipids. Int J Pharm 2003; 258: 133-139.

95- Verma DD, Verma S, Blume G, Fahr A. Particle size of liposomes influences dermal delivery of substance into skin. Int J Pharm 2003; 258: 141-151.

96- Trotta M, Peira E, Debernardi F, Gallarate M. Elastic liposomes for skin delivery of dipotassium glycyrrhizinate. Int J Pharm 2002; 241: 319-327.

97-Cevc G, Schätzlein A, Richardsen H. Ultradeformable lipid vesicles can penetrate skin and other semi-permeable membrane barriers unfragmented. Evidence from double label CLSM experiments and direct size measurement. Biochim Biophys Acta 2002; 1564: 21-30.

98-Cevc G, Gebauer D. Hydration-driven transport of deformable lipid vesicles through fine pores and skin barrier. Biophys J 2003; 84: 1010-1024.

99- Honeywell-Nguyen PL, Wouter Groenink HW, de Graaff AM, Bouwstra JA. The in vivo transport of elastic vesicles into human skin: effects of occlusion, volume and duration of application. J Control Rel 2003; 90(2): 243-255.

100- Schramlova J, Blazek K, Bartackova M, Otova B, Mardesicova L, Zizkovsky V, Hulinska D. Electron microscopic demonstration of the penetration of liposomes through skin. Folia Biol 1997; 43(4):165-169.

101- El Maghraby GM, Williams AC, Barry BW. Skin hydration and possible shunt route penetration in controlled skin delivery of estradiol from 
ultradeformable and standard liposomes in vitro. J Pharm Pharmacol 2001; 53: 1311-1322.

102- $\quad$ Han I, Kim M, Kim J. Enhanced transfollicular delivery of adriamycin with a liposome and iontophoresis. Exp Dermatol 2004; 13(2): 86-92.

103- Cevc G, Blume G, Schätzlein A, Gebauer D, Paul A. The skin: a pathway for systemic treatment with patches and lipid-based agent carriers. Advanced Drug Del Rev. 1996; 18: 349-378. 


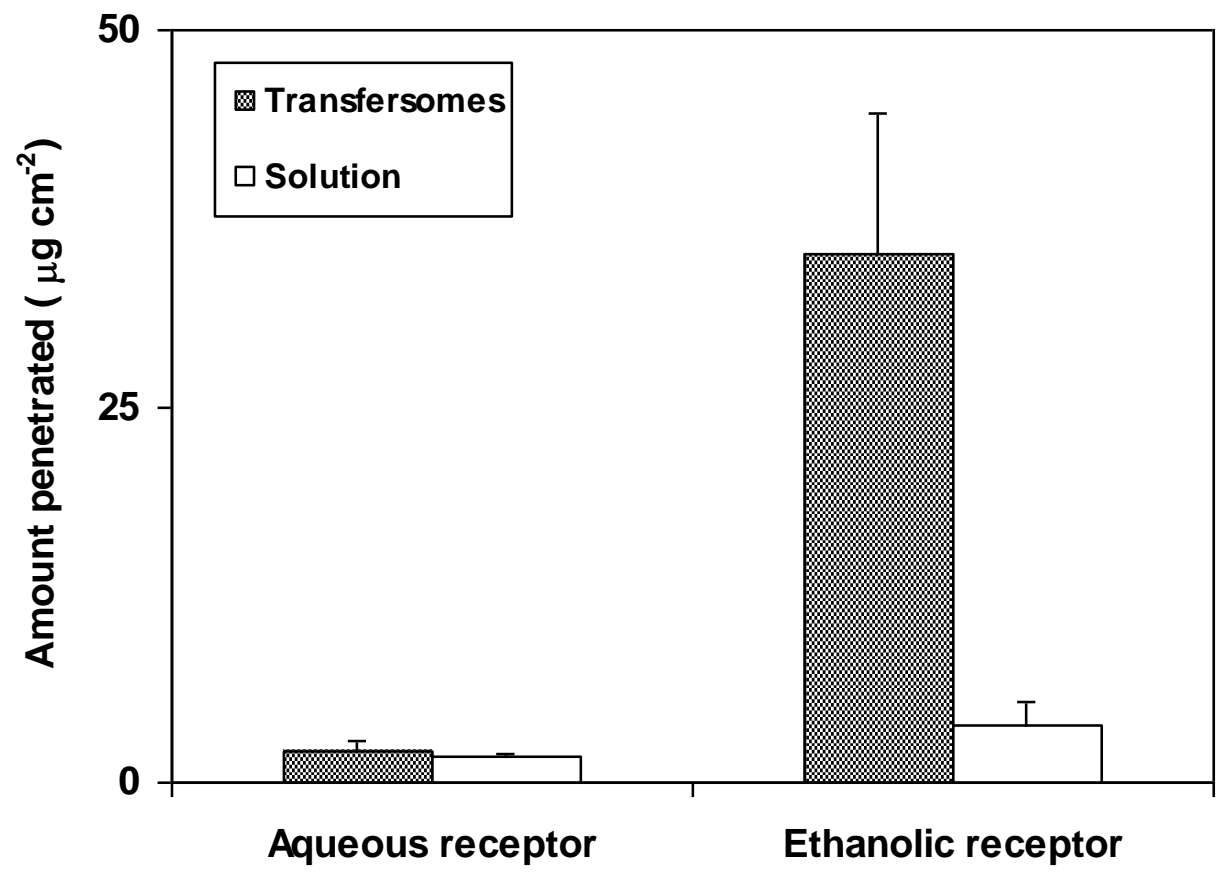

Fig. 1 Amount of 5-fluorouracil penetrated through human epidermis 36 hours after application of Transfersomes or saturated aqueous solution. 

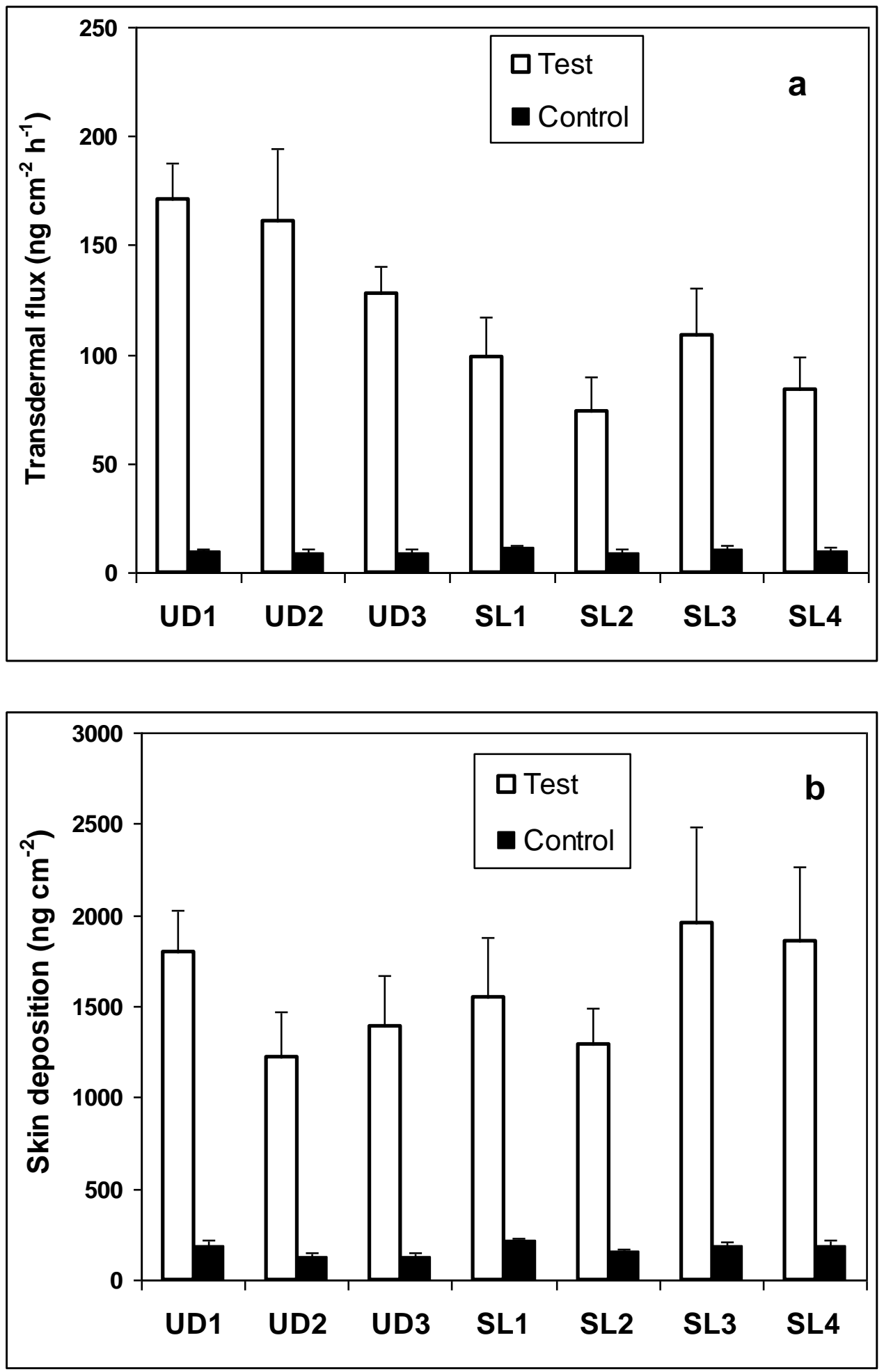

Fig.2 Transdemal flux (a) and skin deposition (b) of estradiol obtained after open application of various vesicular formulations or saturated aqueous solution (control). 


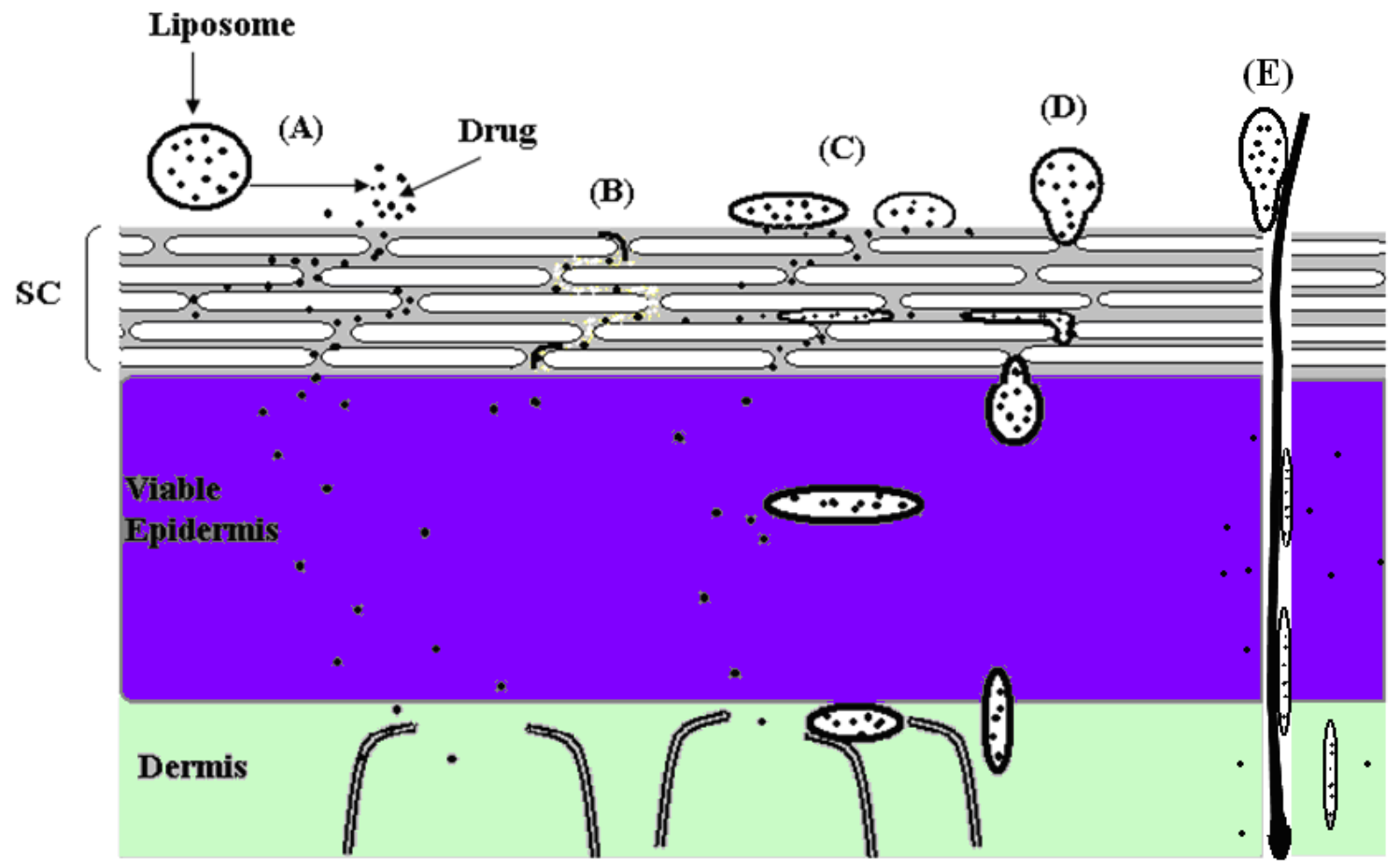

Fig. 3 Possible mechanisms of action of liposomes as skin drug delivery systems. (A) is the free drug mechanism, (B) is the penetration enhancing process of liposome components, (C) indicates vesicle adsorption to and/or fusion with the stratum corneum (SC), (D) illustrates intact vesicle penetration into or into and through the intact skin and (E) shows delivery through an appendage (not to scale) (modified from reference [15]). 


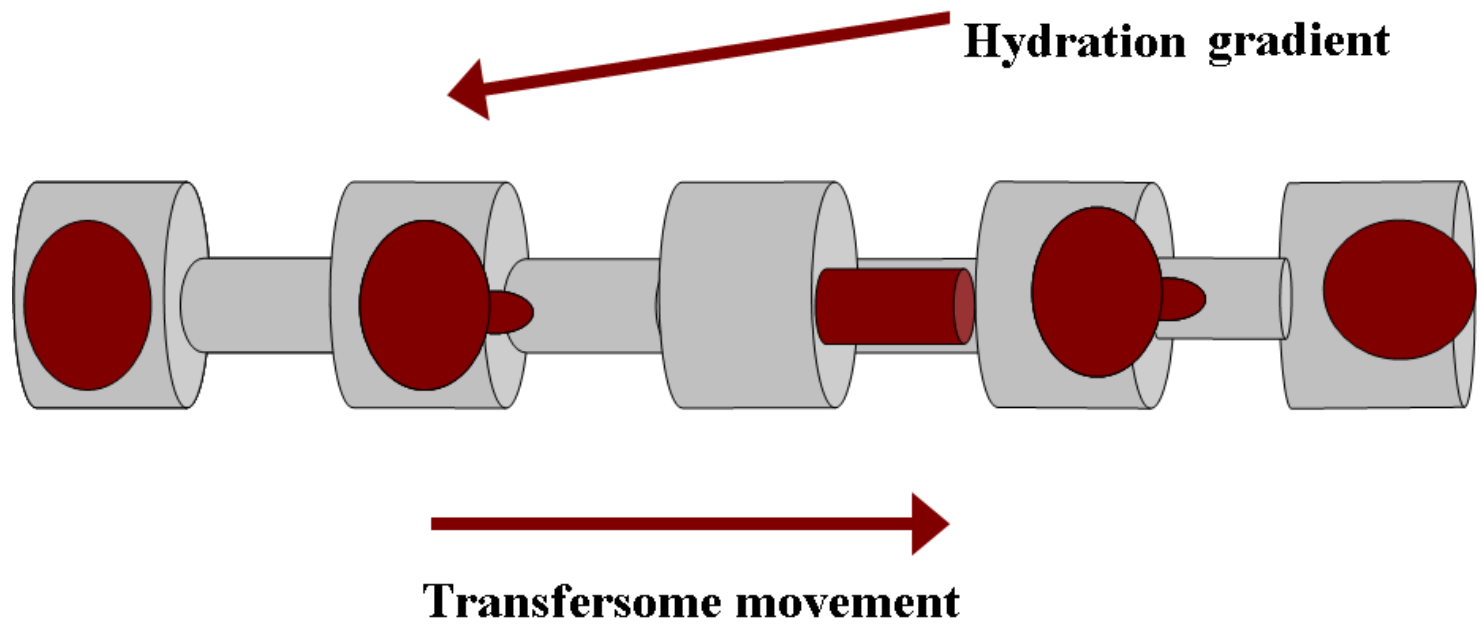

Fig. 4 Hydration gradient and deformability-driven movement of Transfersomes through small pores (after Cevc et al., 1996) [103]. 